\title{
Anal Neuroendocrine Tumor Masquerading as External Hemorrhoids: A Case Report
}

\author{
Muhammad Khan ${ }^{\mathrm{a}}$, Ahmed Dirweesh ${ }^{\mathrm{a}, \mathrm{c}}$, Chikezie Alvarez ${ }^{\mathrm{a}}$, Herbert Conaway ${ }^{\mathrm{a}}$, \\ Robert Moser ${ }^{b}$
}

\begin{abstract}
Neuroendocrine tumors in gastrointestinal (GI) tract are a rare source of GI malignancy with an estimated incidence of 2.5 - 5 per 100,000 people per year and the prevalence of 35 per 100,000. In the GI tract, they are located in decreasing order of frequency in appendix, ileum, rectum, stomach, and colon. Those found in the anal region represent just $1 \%$ of all malignancies of the anal canal. Their clinical presentation can be widely varying, sometimes being found incidentally with metastatic disease and an unknown primary source. We report a case of a 60 -year-old male who presented with a 2-week history of intermittent bright red blood per rectum and anal pain. He was found to have a lesion in the perianal area which was subsequently diagnosed has a poorly differentiated large cell type neuroendocrine carcinoma (NEC) with hepatic metastasis.
\end{abstract}

Keywords: Neuroendocrine; Anus; Rectum

\section{Introduction}

Neuroendocrine tumors (NETs) comprise of a large heterogeneous group of tumors, which have various clinical presentations, and rates of tumor growth. The largest group of these tumors is the gastroenteropancreatic (GEP) NETs. The incidence has been estimated to be 2.5 - 5 per 100,000 people per year with a prevalence of 35 per 100,000 [1]. Various studies have shown a steadily increasing incidence of gastrointestinal (GI) NETs along with increasing survival rates over the past three decades; however, the prognosis for poorly differentiated NETs remains dismal [2].

Tumors of the anal canal are exceedingly rare, accounting for $5 \%$ of anorectal malignancies with NETs comprising $1 \%$ of all tumors of the anal canal [3].

Manuscript accepted for publication January 23, 2017

${ }^{a}$ Department of Internal Medicine, Saint Francis Medical Center, Seton Hall University, Trenton, NJ, USA

bDepartment of Pathology, Saint Francis Medical Center, Trenton, NJ, USA

${ }^{\mathrm{c} C o r r e s p o n d i n g ~ A u t h o r: ~ A h m e d ~ D i r w e e s h, ~ D e p a r t m e n t ~ o f ~ I n t e r n a l ~ M e d i-~}$ cine, Saint Francis Medical Center, Seton Hall University, Trenton, NJ, USA. Email: adirweesh@stfrancismedical.org

\section{Case Report}

A 60 -year-old black male presented with a 2-week history of intermittent bright red blood per rectum and anal pain. Over the past 2 months, he had noticed tenesmus and increased constipation. He denied abdominal pain, nausea, vomiting, anal or perianal discharge or sinuses, fever, chills, anorexia or weight loss. He had a past medical history of hypertension, hyperlipidemia, coronary artery disease status post coronary artery bypass surgery, and peripheral vascular disease status post multiple stents.

On examination, his temperature was $97.8^{\circ} \mathrm{F}$, heart rate was $80 / \mathrm{min}$, respiratory rate was $16 / \mathrm{min}$, BP was $120 / 86 \mathrm{~mm}$ $\mathrm{Hg}$ and oxygen saturation was $98 \%$ on room air. Abdominal examination was grossly normal without tenderness, swellings or hepatosplenomegaly. Rectal examination was significant for a $2 \mathrm{~cm}$ lesion located in his perianal area that mimicked a thrombosed external hemorrhoid. It was slightly painful, and partially covered with anal skin (Fig. 1). The rest of the anorectal examination was normal and inguinal lymph nodes were not palpable.

His CBC showed hemoglobin of $11.2 \mathrm{~g} / \mathrm{dL}$, WBCs of 3

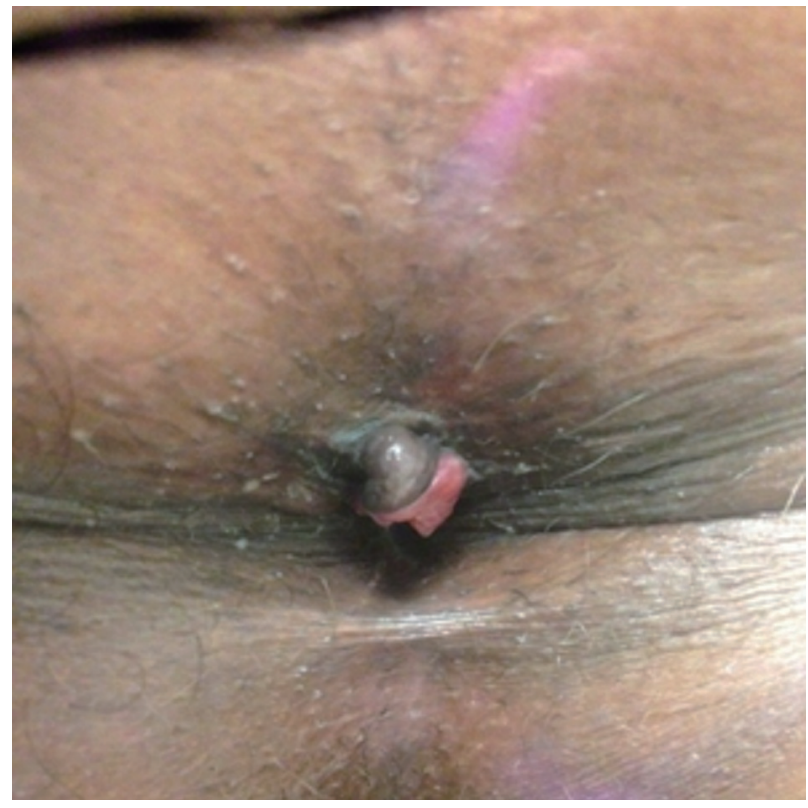

Figure 1. Anal mass partially covered with skin. 


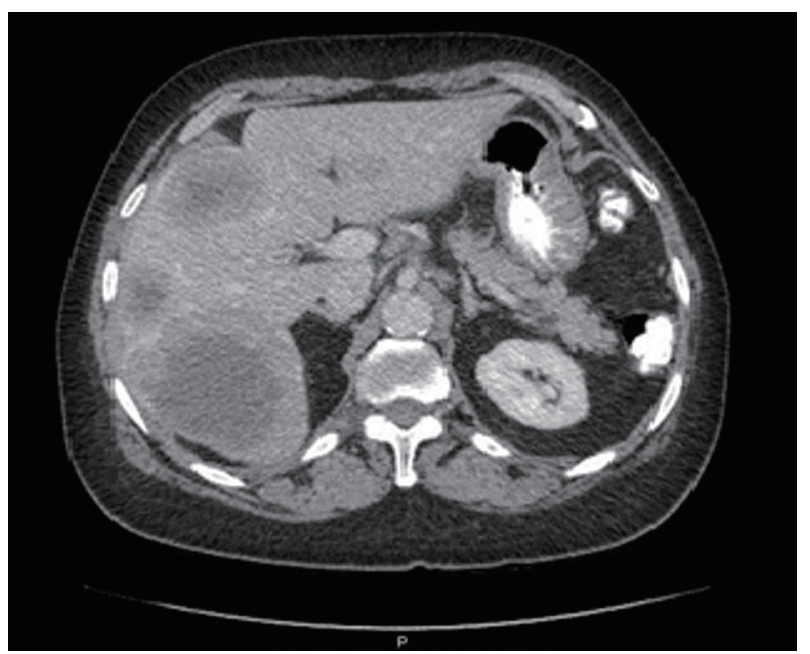

Figure 2. A contrast-enhanced CT scan of the abdomen showing multiple hepatic lesions, consistent with metastatic disease.

$\times 10^{9} / \mathrm{L}$, and platelets of $184 \times 10^{9} / \mathrm{L}$. His creatinine was 0.60 $\mathrm{mg} / \mathrm{dL}$ and blood urea nitrogen was $12 \mathrm{mg} / \mathrm{dL}$.

A contrast-enhanced CT scan of the chest, abdomen and pelvis showed multiple large hepatic mass lesions, consistent with hepatic metastatic disease (Fig. 2).

Excisional biopsy of the anal lesion revealed an invasive high-grade neuroendocrine carcinoma (NEC) of a large cell type (Fig. 3a, b).

Immunohistochemical studies were positive for cytokeratin, chromogranin, and synaptophysin. There was a high Ki-67 proliferation index of $>90 \%$. The patient started a chemotherapy-based regimen and was currently getting his scheduled cycles with resolution of the clinical symptoms and regression of the hepatic lesions.

\section{Discussion}

The most common sites of NETs in the GI tract include the small intestine followed by the pancreas [1]. Anal NETs are exceedingly rare, accounting for $1 \%$ of the anal canal malig- nancies.

NETs share several common features on histological and pathological examination. On histological examination, NETs show clusters or ribbons of round to fusiform small to intermediate-sized cells with variably abundant mitoses represent NECs [4]. On immunohistochemical staining, they are also positive for chromogranin, synaptophysin or neuron-specific enolase. In our patient, immunohistochemical studies were positive for cytokeratin, chromogranin, and synaptophysin. NETs are also neuron-specific enolase (NSE) and CD56 is often positive, but is non-specific $[5,6]$.

There are several existing systems, which attempt to histologically and anatomically classify neuroendocrine neoplasms (NENs). The two main systems adopted are the World Health Organization (WHO) and the European Neuroendocrine Tumor Society (ENETS). Both of these systems classify NENs on the basis of the grade of the tumor, which is assessed by the mitotic rate and Ki-67 index. According to the WHO 2010/ENETS, NENs are broadly classified as well-differentiated NENs and poorly differentiated NEC [5]. Well-differentiated NETs are subdivided into low grade (G1) and intermediate grade (G2), whereas high-grade NECs (G3) are subdivided into small cell carcinoma and large cell NEC [5]. Immunohistochemistry for Ki-67 (MIB-1) as well as the mitotic count is mandatory to grade the tumor. Well-differentiated NETs carry a mitotic count of less than 20 per 10 high-power field (HPF) and a Ki-67 index greater than $20 \%$ [5]. High-grade NETs have a mitotic count of greater than 20 per $10 \mathrm{HPF}$ and a Ki-67 index more than $20 \%$ [5]. NENs carry poor prognosis with one-third of patients having distant metastasis at the time of presentation most commonly involving lymph nodes, liver and bones [5]. Our patient had a Ki-67 index greater than 90\% and an excisional biopsy which revealed an invasive high-grade NEC of a large cell type. Large tumor size, lymphovascular invasion, high mitotic rate and high Ki-67 index are poor prognostic markers.

Imaging whether it be CT scan, MRI, ultrasound, positron emission tomography (PET), somatostatin receptor scintigraphy or even a multimodality approach is necessary, when trying to detect an unknown primary NENs, and also aids the staging and treatment process. Despite these various modalities, in $20-50 \%$ of cases of NENs, the primary tumor site remains unknown [5]. CT scan allows the primary tumor to be
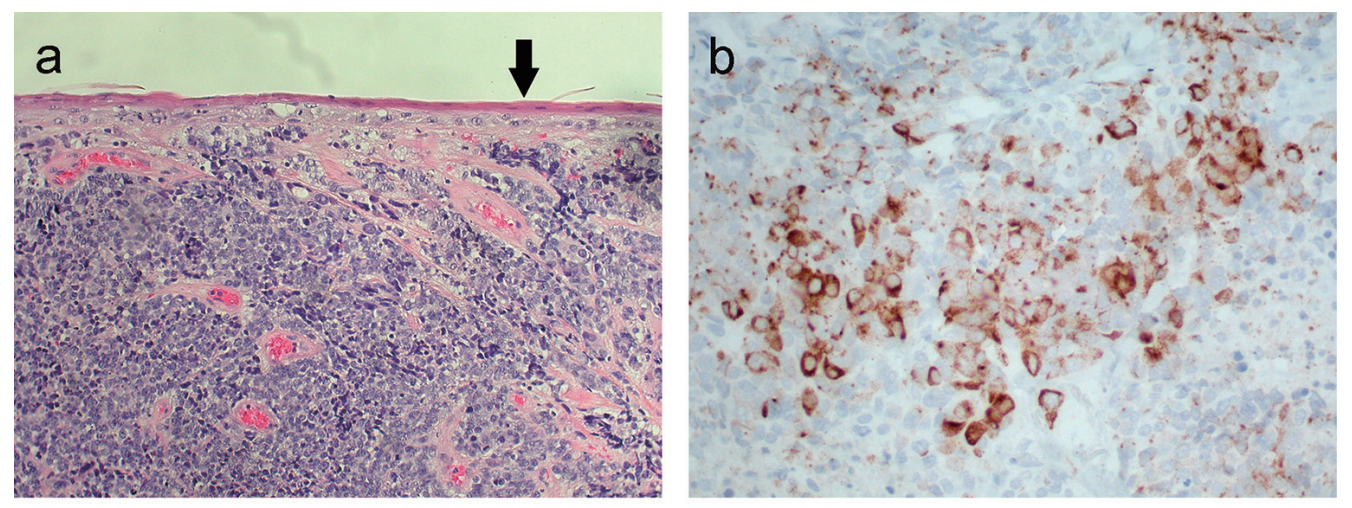

Figure 3. (a) High-grade neuroendocrine carcinoma invading beneath the anal squamous epithelium (arrow). (b) Positive immunohistochemical brown staining of tumor cell cytoplasm (chromogranin, $\times 400$ ). 
located in $22-45 \%$ of patients. Endoscopic ultrasound (EUS) has a greater sensitivity and specificity than trans-abdominal ultrasound (TAUS) in detecting pancreatic NENs [7]. MRI is considered to be superior to CT scan for lesion assessment of the visceral solid organs as one study showed superiority of MRI towards CT for the detection of metastatic lesions [7]. Radiologic diagnostic modalities can also be complemented with radionuclide scans such as the somatostatin receptor scintigraphy (octeotide scan) and PET scan using specific isotopes such as $68 \mathrm{Ga}$-DOTA-octreotate [7]. In one prospective study, ${ }^{68} \mathrm{Ga}$-DOTATATE PET/CT was shown to be superior to ${ }^{111} \mathrm{In}$ pentetreotide SPECT/CT and anatomic imaging in the detection of unknown primary tumors and primary and metastatic NENs [8]. In our patient, the diagnostic workup was aided by the external location of his primary tumor.

Clinically, NENs can present with a variety of symptoms, which are largely dependent on the functional status of the tumor, its anatomical location and hormonal secretion product. Insulinoma or VIPoma can present with symptoms of recurrent episodes of hypoglycemia or with watery diarrhea, dehydration and hypokalemia. Functional NENs are more common in the foregut and the hindgut, with non-functional tumors representing the majority of NENs found in the hindgut [9]. Our patient had a very non-functional non-secretory tumor where his symptoms were secondary to the anatomic location of his tumor.

Although the standard therapy for patients with NECs of the anal canal is still unclear, literature shows early diagnosis and surgical intervention are imperative and treatment approach should be directed by age, stage, nodal involvement, and tumor histopathology [10]. Somatostatin analogs such as octreotide are the recommended first line therapy in non-functioning as well as functioning progressive well-differentiated NETs [5]. Currently, the role of surgery in NECs is unclear. A chemotherapy regimen combining etoposide plus cisplatin or carboplatin is currently considered useful for poorly differentiated tumors and has shown reasonable results [5].

\section{Conclusion}

The case highlights the unusual anal location and aggressive nature of high-grade large cell NEC. One must always keep this differential in mind especially when faced with a nonfunctional tumor presenting with vague non-specific symptoms.

\section{Grant Support}

None.

\section{Financial Disclosures}

None.

\section{Conflicts of Interest}

None.

\section{References}

1. Oberg K, Castellano D. Current knowledge on diagnosis and staging of neuroendocrine tumors. Cancer Metastasis Rev. 2011;30(Suppl 1):3-7.

2. Lawrence B, Gustafsson BI, Chan A, Svejda B, Kidd M, Modlin IM. The epidemiology of gastroenteropancreatic neuroendocrine tumors. Endocrinol Metab Clin North Am. 2011;40(1):1-18, vii.

3. Beahrs OH, Wilson SM. Carcinoma of the anus. Ann Surg. 1976;184(4):422-428.

4. Kobayashi H, Ueno H, Hashiguchi Y, Ishiguro M, Omata J, Kajiwara Y, Shimazaki H, et al. T1 neuroendocrine carcinoma of anal canal after transanal resection for intramucosal adenocarcinoma. Jpn J Clin Oncol. 2006;36(5):325328.

5. Oberg K, Knigge U, Kwekkeboom D, Perren A. Neuroendocrine gastro-entero-pancreatic tumors: ESMO Clinical Practice Guidelines for diagnosis, treatment and followup. Ann Oncol. 2012;23(Suppl 7):vii124-130.

6. Klas JV, Rothenberger DA, Wong WD, Madoff RD. Malignant tumors of the anal canal: the spectrum of disease, treatment, and outcomes. Cancer. 1999;85(8):1686-1693.

7. Tan EH, Tan CH. Imaging of gastroenteropancreatic neuroendocrine tumors. World J Clin Oncol. 2011;2(1):2843.

8. Sadowski SM, Neychev V, Millo C, Shih J, Nilubol N, Herscovitch P, Pacak K, et al. Prospective Study of 68GaDOTATATE Positron Emission Tomography/Computed Tomography for Detecting Gastro-Entero-Pancreatic Neuroendocrine Tumors and Unknown Primary Sites. J Clin Oncol. 2016;34(6):588-596.

9. Schott M, Kloppel G, Raffel A, Saleh A, Knoefel WT, Scherbaum WA. Neuroendocrine neoplasms of the gastrointestinal tract. Dtsch Arztebl Int. 2011;108(18):305312.

10. Metildi C, McLemore EC, Tran T, Chang D, Cosman B, Ramamoorthy SL, Saltzstein SL, et al. Incidence and survival patterns of rare anal canal neoplasms using the surveillance epidemiology and end results registry. Am Surg. 2013;79(10):1068-1074. 